\title{
Evidence for cardiovascular autonomic nerve dysfunction in multiple sclerosis
}

\author{
MANOHARA PJ SENARATNE, ${ }^{*}$ DOROTHY CARROLL, KENNETH G WARREN, \\ TISSA KAPPAGODA
}

From the Department of Medicine, University of Alberta Hospital, Edmonton, Alberta, Canada

SUMMARY In 21 patients with multiple sclerosis and 20 healthy controls, the following tests of autonomic function were examined: (1) variation in heart rate during deep breathing, (2) the variations in heart rate and systolic blood pressure during a standardised Valsalva manoeuvre, (3) the changes in heart rate and systolic blood pressure during arm ergometry starting at $30 \mathrm{~W}$ with increments of $20 \mathrm{~W}$ every $3 \mathrm{~min}$. In the control subjects the maximum variation in heart rate from inspiration to expiration was $>16$ beat/min (range 17-43). In patients with multiple sclerosis, five had a maximum variation in heart rate of $<13$ beat/min which was considered as the lower limit of normal. The Valsalva ratio in the control subjects ranged from 1·33-3·24. Four patients had Valsalva ratios of $<1 \cdot 30$. In patients with multiple sclerosis the heart rate and systolic blood pressure responses to exercise were attenuated significantly in four and seven subjects respectively. It is concluded that a significant number of patients with multiple sclerosis show evidence of autonomic dysfunction involving the cardiovascular system.

It is well known that multiple sclerosis can lead to dysfunction of the autonomic nervous system. Thus bladder dysfunction leading to retention of urine and incontinence and sexual dysfunction leading to impotence are relatively common clinical features in patients with this disease. The manifestations of this autonomic nerve dysfunction as it affects the organs of the pelvis, especially the bladder, have been investigated in detail. ${ }^{2}$ However, there are relatively few reports on the cardiovascular manifestations of this condition. ${ }^{34}$

In a study of 50 patients with multiple sclerosis, Cartlidge $^{3}$ reported no evidence of postural hypotension or abnormal blood pressure responses to exercise. In patients at rest, Neubauer and Gundersen ${ }^{4}$ observed a reduction in the variations of the heart rate with respiration, which they attributed to dysfunction of the autonomic control of the heart and circulation. The present investigation was

${ }^{*}$ Research Fellow supported by the Alberta Heritage Foundation for Medical Research

Address for reprint requests: Dr CT Kappagoda, Rm. 2c-244, Division of Cardiology, Walter Mackenzie Health Sciences Centre, University of Alberta, Edmonton, Canada, T6G 2R7.

Received 2 December 1983 and in revised form 6 March 1984 Accepted 10 March 1984 undertaken to examine autonomic regulation of the cardiovascular system in patients with multiple sclerosis using the following tests: (a) the variation in heart rate during deep breathing, (b) the heart rate and blood pressure responses to the Valsalva manoeuvre and, (c) the heart rate and blood pressure responses to arm ergometry.

\section{Subjects and methods}

Twenty healthy subjects (controls) and 21 patients with multiple sclerosis were studied. All the subjects were informed of the aims of the study and agreed to participate in it. The controls were hospital personnel with no known history or symptoms suggestive of diabetes mellitus, cardiovascular or renal disease. There were nine men and 11 women in this group and their ages ranged from 22-51 years (mean $37 \cdot 8$ years). There were 13 men and eight women in the Patient Group. In this latter group the diagnosis of multiple sclerosis had been established on the basis of Schumacher's criteria. ${ }^{5}$ Nineteen patients had been categorised as clinically definite and the other two as clinically probable. Patients admitted to the hospital for short periods of rehabilitation or for treatment of exacerbations of the disease were considered for the study and only those considered well enough to undertake the tests were selected. 
None of the patients or controls had clinical signs of cardiac disease or abnormalities in the electrocardiogram (ECG) at rest. All subjects were rested for ninety minutes prior to the commencement of the tests. During the study, the ECG was recorded using lead CM5 which was connected to an amplifier (Model 127 002/2, Kontron Medical International, Zurich, Switzerland), the output of which was displayed on an oscilloscope (Model 127 002/1, Kontron Medical International, Zurich, Switzerland) which incorporated a cardiotachometer. In addition, the respiratory movements were recorded from the same electrocardiographic leads (Model 127 002/2, Kontron Medical International, Zurich, Switzerland). An analogue output from the cardiotachometer and the tracing of the respiratory movements were recorded separately (Model 220, Gould Instruments Inc, Cleveland, Ohio, USA) providing a continuous tracing of the heart rate and the excursion of the chest wall respectively. The blood pressure was recorded using a conventional clinical sphygmomanometer.

\section{TESTS OF AUTONOMIC FUNCTION}

(a) Heart rate variation during deep breathing ${ }^{6}$

The subjects were recumbent and the electrocardiogram was recorded for one minute as a control period. Then the subjects commenced breathing deeply at a rate of approximately 6 breaths per minute. The occurrence of adequate chest movements, during the period of deep breathing was confirmed with the help of the recordings (see above). The heart rate was recorded continuously during 8-10 consecutive breaths in each subject. From these recordings the difference between the maximum and minimum heart rates during deep breathing was measured for each respiratory (that is inspiratory-expiratory) cycle. From this data the following "measurements" were determined:

1. The maximum variation in heart rate from inspiration to expiration in any single respiratory cycle (MVHR). A MVHR of less than 13 beats per minute was considered abnormal. ${ }^{\circ}$

2. The average variation in heart rate from inspiration to expiration for all the respiratory cycles during the period of deep breathing (AVHR).

3. The average maximum and minimum heart rates during inspiration and expiration expressed as a ratio (I:E).

$\mathrm{I}: \mathrm{E}=\frac{\text { Mean Value for Maximum Heart Rate During Each Inspiration }}{\text { Mean Value for Minimum Heart Rate During Each Expiration }}$

This ratio is numerically identical to the "E:I ratio" described by Sundkvist $e t$ al $^{7}$ who based their calculations on the maximum and minimum $R-R$ intervals.

\section{(b) Valsalva manoeuvre 89}

The subjects were asked to blow into a mercury manometer and maintain a pressure of $40 \mathrm{~mm} \mathrm{Hg}$ for 15 seconds. The ECG was recorded before, during and for 30 seconds after the manoeuvre. The blood pressure was measured before, during the final 5 seconds of the "strain" and after resumption of normal breathing.

The Valsalva Ratio which is the maximum heart rate during the manoeuvre divided by the minimum heart rate after resuming spontaneous respiration was calculated.

The recordings of both the above tests were taken after a period of "training" during which the subjects familiarised themselves with the apparatus. This training varied from one to several trials depending on the subject. Patients who were unable to perform the tests satisfactorily (because of incoordination, etc.) were not tested. Any tests considered as abnormal were repeated for confirmation.

\section{(c) Arm ergometry}

The subjects were exercised in an electrically braked arm ergometer (Model EO 22 E1852, Siemans Elema). Arm ergometry was adopted as the preferred form of exercise for this study as the majority of the patients with multiple sclerosis were disabled to a lesser degree in their upper limbs than in the lower limbs. The exercise was started with a load of 30 watts (W) and increased by $20 \mathrm{~W}$ every $3 \mathrm{~min}$. It was terminated when the patients were unable to continue the activity without discomfort. The ECG was monitored continuously and the average heart rate during the 3rd minute of each stage of exercise was used in the analysis. The blood pressure was recorded using a sphygmomanometer during the 3 rd minute of each stage. The responses to exercise were assessed by plotting the values of heart rate and systolic blood pressure against the load.

\section{STATISTICAL METHODS ${ }^{\circ}$}

The data from the patient and control groups were compared by means of the Wilcoxan's rank sum test for unpaired data. The Spearman's rank correlation coefficient (rs) was calculated for the correlation analyses. A p value of $<0.05$ was taken as significant. These non-parametric tests were selected in order to avoid the assumption that the data had a normal distribution. The heart rate and systolic blood pressure responses to exercise during arm ergometry were defined by linear regression analysis against the load in watts. As an index of the rate of rise of the systolic blood pressure and heart rate during exercise, the average increases per 20 watt increment in load were calculated for each subject investigated.

\section{Results}

The patients consisted of 13 males and eight females and the mean age was 41.0 years (range $28-58$ ). The mean duration of the disease (from the initial diagnosis) was 11.7 years (SEM 2.2, range $1-25$ years). The neurological state of the patients was assessed formally within a day of the investigation and graded according to the Kurtzke Disability Status Scale (KDSS)." The distribution of the scores were as follows:

$\begin{array}{lrrrrrrr}\text { KDSS } & 1 & 2 & 4 & 5 & 6 & 7 & 8 \\ \text { Number of Patients } & 1 & 3 & 2 & 3 & 10 & 1 & 1\end{array}$

(a) Deep breathing (fig 1)

This part of the study was conducted on 20 control 

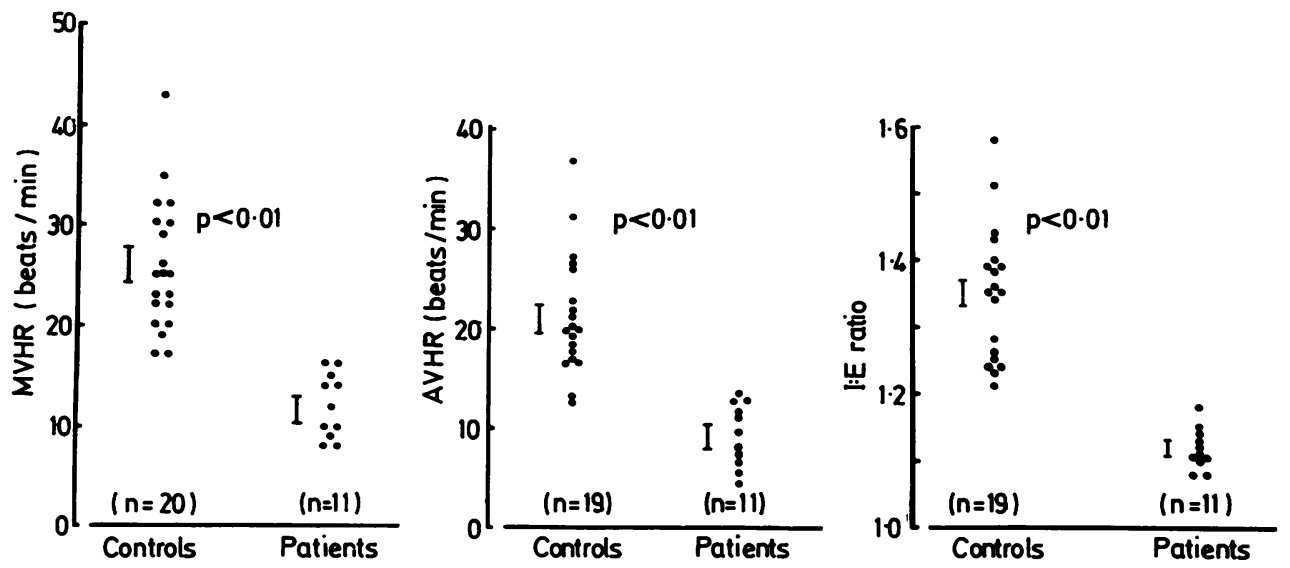

Fig 1 The distributions of the maximum variations in heart rate (MVHR-beat/min), the average variations in heart rate ( $A V H R$ - beat/min) and the I:E ratios during deep beathing in the control subjects and patients. Bars represent mean \pm standard error of mean. MVHR, $A V H R$ and I:E ratio are defined in text. Note: in one control subject the recordings did not permit a reliable assessment of the $A V H R$ and the I:E ratio.

subjects and 11 patients. All the control subjects tested showed sinus arrythmia of varying magnitude during quiet breathing. The variations in heart rate associated with inspiration and expiration increased markedly during deep breathing. The MVHR during deep breathing was 25.7 beat/min (mean; SEM $1 \cdot 5)$. The lowest value observed in these subjects was $17 \mathrm{beat} / \mathrm{min}$. The AVHR in the control subjects was 21.0 beat/min (mean; SEM 1.4; range 12.3$36.5)$. The $I: E$. ratio in these subjects was $1 \cdot 35$ (mean; SEM 0.02; range $1 \cdot 21-1 \cdot 58$ ). Of the three criteria only the AVHR had a significant negative correlation with the age of the control subjects with an rs value of $-0.59(p<0.05)$.

In the 11 patients in whom the deep breathing tests were done the MVHR was 12.0 beat $/ \mathrm{min}$ (mean; SEM 0.9, range 8-16) with six patients having a MVHR of less than 13 beat $/ \mathrm{min}$. The AVHR in these 11 patients was 9.4 beat/min (mean; SEM $0 \cdot 9$, range $4 \cdot 2-13 \cdot 1$ ). The lowest six values of the AVHR were obtained in the six patients with a MVHR of less than 13 beat $/ \mathrm{min}$. The I: $E$ ratio in the patients was $1 \cdot 12$ (mean; SEM $0 \cdot 01$, range $1 \cdot 07-$ $1 \cdot 18)$.

Of the three criteria examined, the I:E ratio and the AVHR had significant negative correlations with the age of the patients with rs values of -0.61 and -0.53 respectively $(p<0.05)$. [Three of the six patients who had values which were considered to be low on the deep breathing test were in their fifties. Even though the values in the Patient Group had a significant negative correlation with age, the values obtained in the three control subjects who were in their fifties were considerably greater than those observed in the three patients in this age group: MVHR: 17, 19 and 30 beat $/ \mathrm{min}$, AVHR $12.3,16.4$ and 18.9 beats/min and I:E ratio: 1.23 , $1 \cdot 24$ and $1 \cdot 39]$.

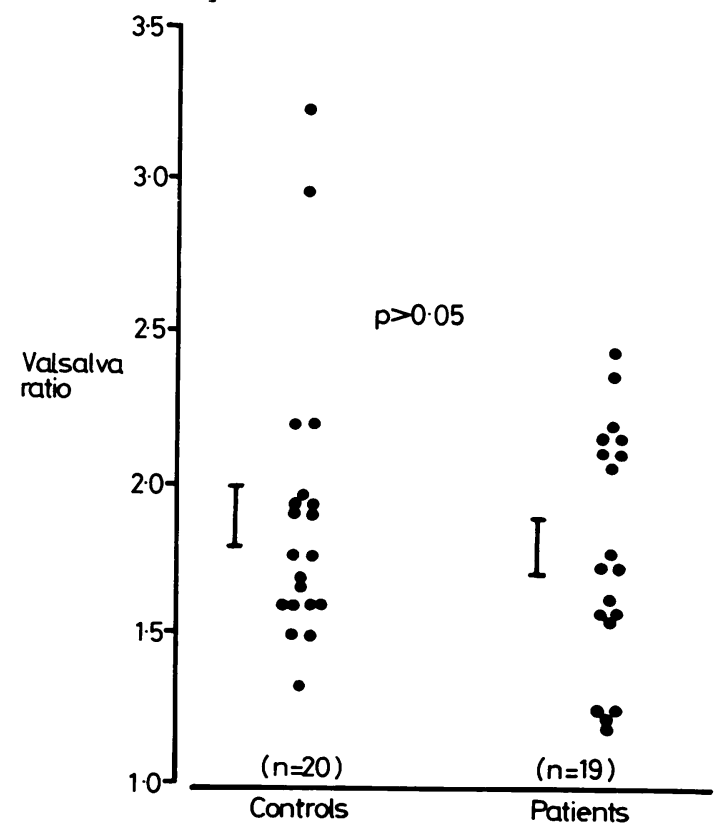

Fig 2 The distribution of the Valsalva ratios during the manoeuvre in the control subjects and in patients. Bars represent mean \pm standard error of mean. Valsalva ratio defined in text. 

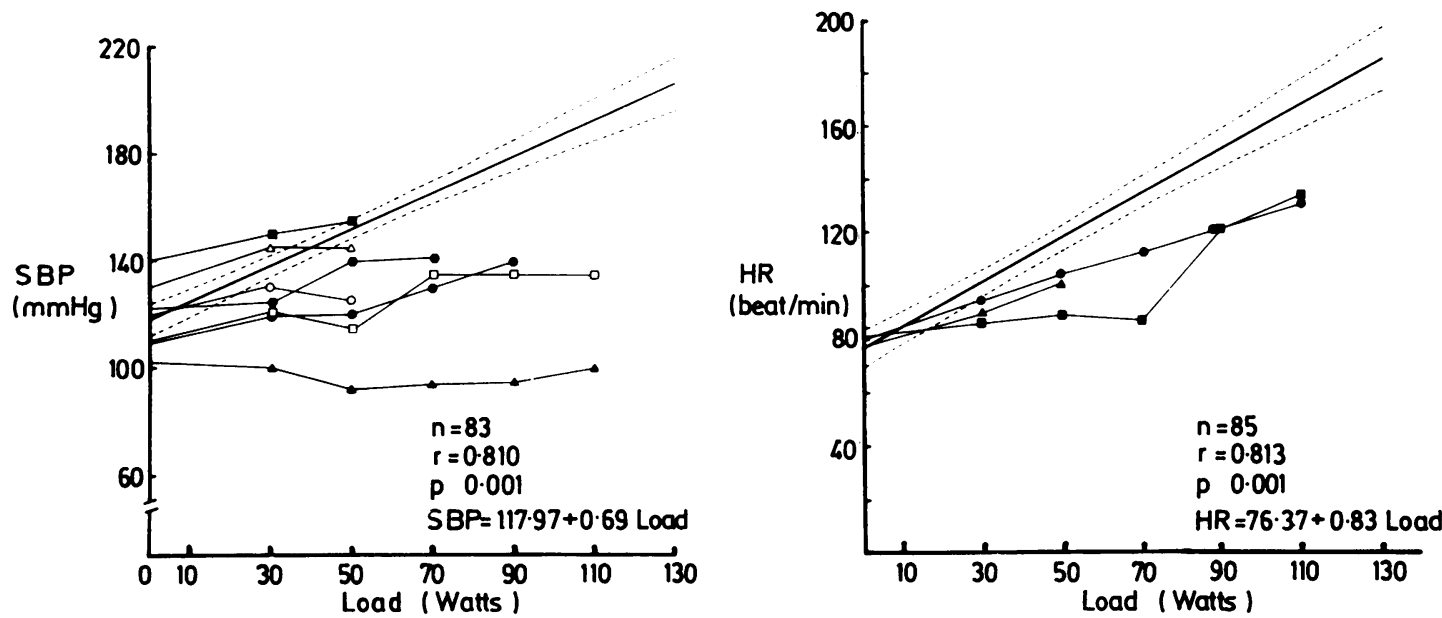

Fig 3 The regression analysis for the systolic blood pressure ( $\mathrm{SBP}-\mathrm{mmHg}$ ) and heart rate (heart rate-beat/min) responses to exercise. Interrupted lines represent the $95 \%$ confidence intervals. Examples of systolic blood pressure and heart rate responses of patients who were considered as abnormal are shown (refer text for explanation).

Also none of the three criteria was correlated significantly with the duration of the illness $(p>$ $0 \cdot 05)$. The data for the 11 patients are summarised in fig 1 . As a group the patients were significantly different from the controls in all three criteria tested $(\mathrm{p}<0.01)$.

(b) Valsalva manoeuvre (fig 2)

All the control subjects tested showed a characteristic response to the Valsalva manoeuvre with a drop in systolic blood pressure and a tachycardia during the period of strain and an increase in systolic blood pressure above control levels with a reflex bradycardia after resumption of normal respiration. In the control subjects, the Valsalva ratio was greater than 1.3 (range $1 \cdot 33-3 \cdot 24$ ). Of the 19 patients tested, four had a Valsalva ratio of 1.3 or less. Three of these patients had no overshoot in the systolic blood pressure after resumption of normal respiration. Of the six patients who had abnormal responses to deep breathing only one had a Valsalva ratio of less than $1 \cdot 3$. There was no significant correlation between the Valsalva ratio and the AVHR, the MVHR or the I:E ratio $(p>0.05)$. The Valsalva ratios of the patients were not significantly different from those of the controls $(p>0.05)$.

\section{(c) Arm ergometry}

The 20 control subjects exercised on the arm ergometer until they were unable to continue without distress. The maximum load achieved had a mode of $70 \mathrm{~W}$ ( 10 subjects) with a range from 50 $130 \mathrm{~W}$. The heart rate and systolic blood pressure rose in all subjects with exercise. Both the heart rate and systolic blood pressure had a significant correlation $(p<0.001)$ with the load (correlation coefficients +0.81 and +0.81 respectively). These results are summarised in fig 3 . It was found that in the control group $(n=20)$, the minimum increase in systolic blood pressure and heart rate per $20 \mathrm{~W}$ increments in load, were $8.9 \mathrm{~mm} \mathrm{Hg}$ and 12 beat min respectively (fig 4).

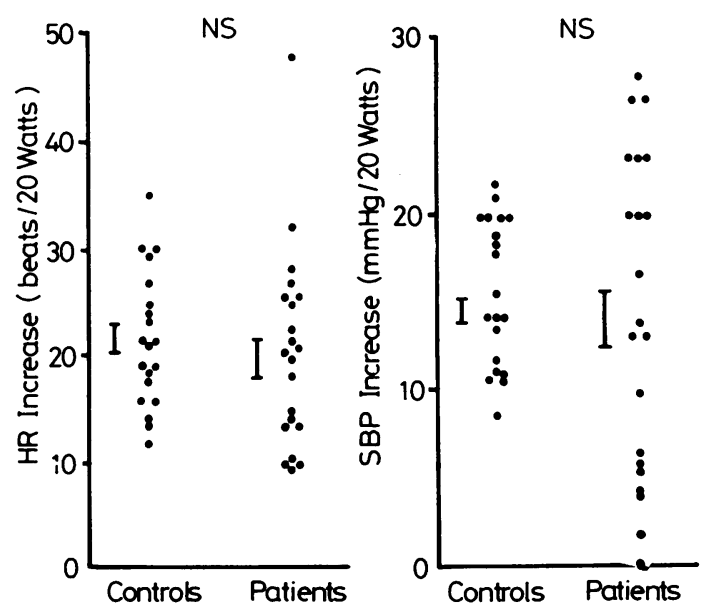

Fig 4 The distributions of the heart rate (beat/min) and systolic blood pressure $(\mathrm{mm} \mathrm{Hg}$ ) increases during exercise per 20 watt increments in load in the control subjects $(n=$ $20)$ and in patients $(n=21)$. Bars represent mean \pm standard error of mean. NS-no significant difference. 
The maximum load achieved by the 21 patients with multiple sclerosis had a mode of $70 \mathrm{~W}$ (seven subjects) with a range of $30-110 \mathrm{~W}$. Five patients were able to complete the first stage of exercise only. Seven patients failed to achieve the minimum increase in systolic blood pressure (in the control subjects) with a $20 \mathrm{~W}$ increment in load, and four patients failed to achieve the corresponding increase in heart rate (fig 4). Three of the latter group also failed to increase their blood pressure during exercise. Examples of responses that were considered as abnormal are shown in fig 3 . None of the patients with multiple sclerosis experienced any adverse changes in their clinical status as a result of the exercise performed for the study. Of the six patients who had an MVHR of less than 13 beat/min, the systolic blood pressure response to exercise was abnormal in two and the heart rate in one.

One patient (BS) had two arm ergometry tests during two clinically different relapses with a gap of 3 months in between. The subject was recovering from a relapse with predominantly brain stem manifestations when he was tested initially. The systolic blood pressure and heart rate responses to exercise were clearly attenuated (changes in systolic blood pressure and heart rate per $20 \mathrm{~W}$ increment in load were $-0.4 \mathrm{~mm} \mathrm{Hg}$ and +9.6 beat $/ \mathrm{min}$ respectively) although the patient was able to exercise up to 110 watts at this time. After a relatively complete remission of 3 months he was re-admitted with weakness in his limbs with no brain stem signs. Both systolic blood pressure and heart rate responses to exercise were within normal limits at this time with $11.4 \mathrm{~mm} \mathrm{Hg}$ and $15.4 \mathrm{beat} / \mathrm{min}$ increases per $20 \mathrm{~W}$ increment in load. Apart from this interesting observation no other correlation was noted between the presence of abnormalities in the tests performed and the clinical features.

\section{Discussion}

It is accepted generally that the Valsalva manoeuvre, and the demonstration of sinus arrhythmia are two simple procedures for establishing the functional integrity of the autonomic innervation of the cardiovascular system. These tests have been used extensively in a variety of clinical situations, particularly in identifying patients with diabetic autonomic neuropathy. ${ }^{67}$ However, there are hardly any reports on the use of these tests in patients with multiple sclerosis. The findings of the study reported here have indicated that a significant proportion of these patients with multiple sclerosis have evidence of autonomic dysfunction affecting the cardiovascular system as revealed by these procedures.

The primary pathological lesions in multiple sclerosis are limited to the central nervous system. Thus, the autonomic disturbances revealed by the Valsalva manoeuvre and by deep breathing are likely to be the results of derangements in the central connections of reflex pathways. The neurons regulating cardiac and respiratory function are located in the periventricular region of the fourth ventricle, which is a common site for plaques in multiple sclerosis. ${ }^{12}$ However, the present study did not demonstrate any correlations between the existence of these abnormalities and the location of the lesions as indicated by the clinical findings. The lack of correlation between the abnormalities observed in the three tests carried out, is not unexpected in view of the patchy nature of the lesions in multiple sclerosis. In addition, it was found that all the patients who had an abnormal sinus arrhythmia did not have also an abnormal Valsalva response. This observation suggests that more than one reflex pathway is involved in the two effects. For instance, in the Valsalva response, the bradycardia in particular, is likely to depend primarily upon the integrity of the baroceptor pathways. The changes in heart rate caused by deep breathing could be attributed to a variety of factors. The conventional view is that the activity of cardiovascular neurons in the medulla is influenced by the activity of the respiratory centres. Alternatively, phasic changes in venous return induced by deep breathing could result in changes in right atrial pressure which in turn could activate the atrial receptors ${ }^{13}$ or stimulate the sinu atrial node directly. ${ }^{14}$ In addition, it is conceivable that "the Group IV afferents" from the respiratory muscles could also make a contribution to this phenomenon. ${ }^{15}$ (See below).

The findings obtained during exercise present an interesting area for speculation. It is apparent that a significant proportion of the patients examined revealed an attenuation of the heart rate and blood pressure responses to exercise. These effects could be viewed as yet another manifestation of a disturbance in the autonomic innervation. Hilsted $^{16}{ }^{17}$ has reported abnormalities in the blood pressure and heart rate responses to exercise in patients with autonomic dysfunction of diabetic aetiology. These changes were associated with a reduction in the catecholamine responses to exercise also. The findings in the present study in relation to arm ergometry could be explained by a similar phenomenon.

However, there is an alternative possibility which should be considered. Longhurst and Mitchell' ${ }^{15}$ have reviewed recently the role of afferent impulses originating from skeletal muscles in the regulation of the heart rate and blood pressure during exercise. The "Group IV afferents" are non-myelinated 
nerves which appear to respond to metabolites generated in skeletal muscle during exercise. Activation of these fibres results in a reflex tachycardia and an increase in blood pressure. Whilst the precise central connections of these fibres remains in doubt, it is widely believed that these afferent impulses are conveyed to higher centres in the posterior columns of the spinal cord. As lesions in the posterior columns are frequent in multiple sclerosis, it is possible, that in some patients with this disease, a disturbance of this particular afferent pathway could result in an abnormal cardiovascular response to exercise.

In conclusion, this study has shown that a significant proportion of patients with multiple sclerosis have evidence of autonomic dysfunction affecting the cardiovascular system. These abnormalities could be demonstrated by the simple procedures described here. Apart from the one example described in this paper, there appears to be no definitive information available about the evolution of these abnormalities. Thus, follow-up investigations are being undertaken on this group of patients for the purpose of obtaining this information. It is conceivable that like the other clinical manifestations of multiple sclerosis, these signs of autonomic dysfunction also could alter in their severity with time.

The authors acknowledge the technical assistance of Mrs Paula Priest and the Alberta Heritage Foundation for Medical Research for financial support.

\section{References}

' Blaivas JG, Bhimani G, Labib KB. Vesico-urethral dysfunction in multiple sclerosis. J Urol 1979;122:342-7.

${ }^{2}$ Bradley WE. Urinary bladder dysfunction in multiple sclerosis. Neurology (Minneap) 1978;28:52-8.

${ }^{3}$ Cartlidge NEF. Autonomic function in multiple sclerosis. Brain 1972;95:661-4.

${ }^{4}$ Neubauer B, Gundersen JG. Analysis of heart rate variations in patients with multiple sclerosis. J Neurol Neurosurg Psychiatry 1978;41:417-9. s Schumacher GA, Beebes G, Kibler RF, et al. Problems of experimental trials of therapy in multiple sclerosis: Report by the panel on the evaluation of experimental trials in multiple sclerosis. Ann NY Acad Sci 1965; 122: 522-68.

- Wheeler T, Watkins PJ. Cardiac denervation in diabetes. Br Med J 1973;4:584-6.

' Sundkvist G, Almer L, Lilja B. Respiratory influence on heart rate in diabetes mellitus. $\mathrm{Br}$ Med $\mathrm{J} \mathrm{1979}$; 1:924-5.

${ }^{8}$ Bennett T, Farquhar IK, Hosking DJ, Hampton JR. Assessment of methods for estimating autonomic nervous control of the heart in patients with diabetes mellitus. Diabetes 1978;27:1167-74.

${ }^{9}$ Zoccali C, Ciccarelli M, Maggiore Q. Defective reflex control of heart rate in dialysis patients: evidence for an afferent autonomic lesion. Clin Sci 1982;63:28592.

${ }^{10}$ Snedecor GW, Cochran WG. Statistical Methods. 6th ed. Ames, Iowa: Iowa State University Press, 1967: $128,135,193$.

"Kurtzke JF. On the evaluation of disability in multiple sclerosis. Neurology (Minneap) 1961;11:686-94.

${ }^{12}$ Oppenheimer DR. Demyelinating diseases. In: Blackwood W, Corsellis JAN, eds. Greenfield's Neuropathology. London: Edward Arnold, 1976: 470-99.

${ }^{13}$ Kappagoda CT, Linden RJ, Snow HM. The effect of stretching the superior venal caval-right atrial junction on right atrial receptors in the dog. $J$ Physiol (Lond) 1972;227:857-87.

${ }^{14}$ Pathak CL. Effects of changes in intraluminal pressure on isotrophic and chromotropic responses of isolated mammalian hearts. Am J Physiol 1958;194:197-9.

is Longhurst JC, Mitchell JH. Reflex control of the circulation by afferents from skeletal muscle. In: Guyton AC, Young DB, eds. International Review of Physiology, Cardiovascular Physiology III, Vol 18. Baltimore: University Park Press, 1979:125-48.

${ }^{16}$ Hilsted J. Pathophysiology in diabetic autonomic neuropathy: cardiovascular hormonal and metabolic studies. Diabetes 1982;31:730-7.

${ }^{17}$ Hilsted J, Galbo H, Christensen NJ. Impaired responses of catecholamines, growth hormone, and cortisol to graded exercise in diabetic autonomic neuropathy. Diabetes 1980;29:257-62. 